\title{
Perspectivas de la cooperación entre América Latina y los países de ASEAN*
}

Francisco Orrego Vicuña**

\section{INTRODUGCIÓN}

Las relaciones entre los países de América Latina y los miembros de la Asociación de Naciones del Sudeste Asiático (ASEAN), han venido intensificándose en los últimos años, dando lugar a una tendencia que tiene ya las caracteristicas de ser muy significativa. No todos Ios países latinoamericanos participan de este proceso, razón por la cual el presente artículo se limitará al caso de los más significativos, destacanđó entre ellos la política de vinculación seguida por Chile, que és la más avanzada en este plano. Por otra parte, en algunas materias será también necesario incluir referencias a otras naciones asiáticas -especialmente Corea, Hong-Kong y Taiwán-, que han surgido igualmente como importantes socios comerciales de América Latina.

Interesa destacar que este proceso de cooperación no se restringe únicamente al campo del intercambio comercial, pues también encuentra expresiones importantes en lo financiero, en la política de recursos, en áreas funcionales y en determinadas situaciones políticas contemporáneas. Los principales aspectos de esta cooperación serán analizados a continuación, incluyendo algunos rasgos de sus antecedentes históricos más relevantes.

\section{ANTECEDENTES HISTÓRICOS DE LA COOPERAGIÓN}

Los primeros contactos modernos entre estas dos regiones geográficas provienen del período de las exploraciones europeas en el Océa-

*Este artículo está basado en una Conferencia que dictó el autor en la Universidad Nacional de Singapur, por invitación del Instituto de Estudios del Sudeste Asiático y del Instituto de Asuntos Internacionales de Singapur, el 10 de noviembre de 1981 . Chile.

**Director del Instituto de Estudios Internacionales de Ia Universidad de 
Francisco Orrego / Perspectivas de la cooperación entíe América Latina...

no Pacífico ${ }^{1}$. En épocas ánteriorès, corrientès migratorias de pueblos asiáticos llegaron hasta las costas americanas a través del Pacífico, principalmente mediante las habilidades de navegación que caracterizaron a los polinésicos, pero ellas no lograron establecer ningún vínculo permanente ni estable Fue el viaje de Magallanes a través del Pacífico (1519-1522) el primer hecho histórico que haría nacer una relación estable. La ruta que siguió este célebre explorador unió por primera vez a Chile con las Filipinas, tocando desde luego en varios puntos del Pacífico Sur. Sólo entonces nació el concepto del Pacífico como una unidad geográfica.

La fuerza expansiva del imperio español de la época fue la que llevó a las primeras formas de intercambio entre estos dos continentes. La expedición de Legazpi se inició en puertos mexicanos en 1564 y condujo a la ocupación de las Filipinas, habiéndosela comparado con gestas tan importantes como el descubrimiento de América por Cristóbal Colón. Poco tiempo después se iniciaba el trayecto del Galeón de Manila a través del Pacífico, que durante dos siglos y medio, entre 1565 y 1815 , condujo comercio y pasajeros entre Manila y el puerto de Veracruz ${ }^{2}$. Estos puertos sirvieron de centros regionales de concentración y distribución de las mercaderías que se intercambiaban a través del océano común, extendiéndose desde allí a los principales puntos de las respectivas regiones.

Con la desaparición del imperio español, cesaron los subsidios y apoyos gubernamentales que habian hecho posible esta vinculación. la cual comenzó a decaer ${ }^{3}$. El último galeón de Manila llegó a América en 1815. Sin embargo, la perspectiva del comercio con Asia logró consolidarse en Chile, país que desde su Independencia en 1810 mantuvo viva la idea de la cooperación a través del Pacífico. Esta fue relativamente constante con las regiones de Polinesia y Micronesia, que son geográficamente las más próximas a Chile, donde el comercio llegó a ser activo, y donde el peso chileno circuló durante gran parte del siglo xrx como moneda de pago.

Sin embargo, la iniciativa llegó más lejos ${ }^{4}$. El trigo y los productos agrícolas de Chile abastecieron durante importantes períodos a

IJ. H. Parry: "Spanish America and the Far East: Origins and development of Trans-Pacific Communication and Trade". En Francisco Orrego Vicuña y Gloria Echeverria Ducó: La comunidad del Pacifico en Perspectiva. Vol. 1. Instituto de Estudios Internacionales de la Universidad de Chile. 1980. (En adelante citado como Comunidad del Pacifico).

"Véase William L. Schurz: The Manila Galleon. New York. 1959. Especialmente el mapa relativo a las rutas del Galeón y de los primeros exploradores europeos. Reproducido en Comunidad del Pacifico. p. 72.

${ }^{3}$ Alvaro Jara: "Las conexiones e intercambios americanos con el Oriente bajo el marco imperial español (siglos xvr"xvin". En Comunidad del Pacifico. Vol. 1.

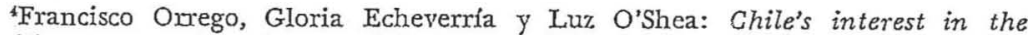
Pacific: an overwiew. A ser publicado por el Instituto de Estudios Internacionales de la Universidad de Chile, 1982. 
Australia, como también fue el caso de California. La compañía Galcutta fue formada en Valparaíso para promover el comercio entre Chile y la India, a través deI Pacífico y eI Indico. Estas y otras empresas no lograrían sobrevivir debido a sus costos y a las dificuItades del transporte. Varios tratados celebrados por Chile en el siglo Xxx, especialmente con Inglaterra, se fundamentaron en el propósito de promover el comercio con las regiones de Asia y el Pacífico, que se encontraban bajo el dominio del Imperio Británico.

Hacia fines del siglo pasado las iniciativas chilenas en el Pacifico comenzaron a disminuir dramáticamente, para Ilegar prácticamente a desaparecer en el siglo xx. Con todo, la presencia intelectual del Pacífico siempre se mantuvo presente, como puede apreciarse en importantes expresiones de la literatura chilena, y otros hechos esporádicos. La anexión de la Isla de Pascua en 1888 contribuyó poderosamente a mantener viva la idea del Pacífico en las décadas que siguieron. Los últimos diez años han visto un renacer de esta política, en la cual la contribución académica ha sido decisiviva, según se examinará más adelante. Este renacimiento no sólo ha tenido lugar en Chile sino que también en otros países latinoamericanos, aspecto que se analizará a continuación.

\section{Garagterústicas generates de las dos regiones}

Es interesante comprobar que América Latina y ASEAN comparten algunas características comunes, como también algunas diferencias importantes, todo lo cual tiene por cierto incidencia en las modalidades de cooperación que se adopten.

La primera realidad que es necesario tener presente es de carácter geográfico. Si se observa un mapa centrado en el Pacífico, se podrá apreciar que a pesar del hecho de que América Latina y ASEAN se encuentran exactamente en el lugar opuesto del mundo, también se encuentran unidos por el Océano Pacífico. La visión tradicional del mundo, que era eurocéntrica, determinaba que fuese imposible pensar en la eventual cercanía del sudeste asiático y el continente sudamericano, pues cada uno se relacionaba principalmente con las respectivas metrópolis europeas. Esta relación ha cambiado hoy día, no porque haya cambiado la geografía, sino porque ha cambiado la manera de apreciar la geografía. Desde este punto de vista, puede sostenerse que hoy día las dos regiones se enfrentan a través del Pacífico, en circunstancias que antes se daban la espalda.

Otras características comunes surgen del examen de algunos indicadores generales. Desde luego, llama la atención el caso de las cifras de población. Mientras ASEAN se acerca a los 300 millones de habitantes, América Latina los sobrepasa ligeramente. En el caso de algunos países individuales, también es posible apreciar seme- 
janzas importantes. Por ejemplo, la población de Brasil es comparable a la de Indonesia, con cifras ligeramente superiores a los 120 y 140 millones de habitantes, respectivamente. Malasia es comparable a Chile y Venezuela, como Filipinas y Tailandia lo son a México en este plano. Las tasas de crecimiento poblacional son también semejantes en ciertos casos, apreciándose un promedio que fluctúa entre el 2 y el $3 \%$ anual.

Quizás más importante que lo anterior, son las cifras relativas al crecimiento económico en las dos regiones. Hasta mediados de la década de 1970, se apreciaban tasas de crecimientos en cierto modo comunes entre los países en desarrollo, con cifras que fluctuaban para ASEAN entre el 2 y el $5 \%$, con algunas excepciones como Singapur, que tradicionalmente ha mostrado un crecimiento superior ${ }^{5}$. En América Latina los promedios eran algo más altos, fluctuando entre un 3 y un $7 \%$ y también exhibiendo algunas cifras más elevadas, particularmente en el caso del Brasil, durante ese período ${ }^{6}$.

A partir de esa fecha comienza a observarse una aceleración del crecimiento de ASEAN, para llegar a promedios actuales en torno al $8 \%$ anual. Si bien en el caso latinoamericano este fenómeno no ha sido tan uniforme, pues se registran disparidades, también se observa el crecimiento acelerado de algunos países, siendo particularmente notorio el caso de Chile, que también ha alcanzado cifras cercanas al $8 \%$ en los últimos años. Ello no se debe a una casualidad, sino que a la aplicación de políticas èconómicas similares, según se examinará en la siguiente sección. Este fenómeno es común con el de otros países en desarrollo avanzados de Asia, cual es el caso de Taiwán, Gorea y Hong-Kong.

Muchos otros indicadores llevarían a establecer comparaciones de interés. Como ejemplo puede mencionarse el control de la inflación en algunos casos de una u otra región, ciertas cifras de comercio exterior -sobre lo que se volverá más adelante- o determinadas comparaciones relativas al Producto Nacional Bruto, entre otras materias. Especialmente interesantes son las cifras del Producto Nacional Bruto de los países más avanzados de Asia -incluyendo a Malasia, Singapur, Taiwán, Corea y Hong-Kong-, y de los más avanzados de América Latina -Argentina, Brasil, Chile, México y Venezuela-, pues los promedios en ambos casos son cercanos a los 1.500 dólares por habitantes.

'Para las cifras generales de ASEAN, véase Jesús P. Estanislao: Towards 1991: An economic entironment in the Pacific Basin with special reference to ASEAN. Pacific Forum Symposium. Bangkok. November 14-15, 1981.

"Para las cifras generales de América Latina, véase Banco Mundial: Informe sobre el Desarrollo Mundial 1981. Agosto de 1981. 


\section{MODELOS ECONÓMICOS Y PERSPECTIVAS DE COOPERACIÓN}

Uno de los factores que más ha estimulado la cooperación entre América Latina y los países miembros de ASEAN, ha sido la similitud de los modelos de desarrollo económico que se han adoptado por varios de los países de cada región, aspecto en el cual nuevamente cabe incluir a los demás países en desarrollo más adelantados de Asia ${ }^{7}$. Si bien por cierto se registran algunas diferencias de énfasis en esta materia, tanto entre las dos regiones como dentro de cada una de ellas, la incorporación de ciertos principios básicos comunes ha determinado una orientación que en lo sustantivo es comparable, aun cuando algunos autores han discutido esta comparación ${ }^{8}$.

La principal característica de estos modelos es la de fundamentar las respectivas economías en el funcionamiento del mercado, reduciendo el margen de intervención del Estado en la economía o bien sujetándolo a claras normas de competencia y eficiencia. La eliminación de los controles sobre precios es una de las consecuencias directas de esta política, permitiendo que el mercado determine este factor. También el estímulo al papel del sector privado es una de las manifestaciones más típicas de esta política, lo que influye en una adecuada movilización de los recursos productivos.

De la misma manera, muchas de estas economías se caracterizan por su importante grado de apertura al comercio internacional, lo que ha permitido tipificarlas como "economías pequeñas y abiertas" en algunos casos. Sobre esta base se han liberalizado en general las operaciones financieras internacionales y se ha adoptado una política de no discriminación respecto de las inversiones extranjeras. A su vez, las políticas monetarias y cambiarias se han liberalizado en otros casos, o bien se han alineado con monedas duras de circulación internacional, principalmente el dólar de los Estados Unidos.

En el ámbito de ASEAN se pueden identificar políticas de esta naturaleza en el caso de Singapur - que es una de las economías más típicas en este plano-, y parcialmente en el de Malasia. Otros de los países de ASEAN comparten algunos aspectos de este enfoque liberal, pero no su totalidad. Igualmente este es el caso de HongKong, Taiwán y Corea, que también son típicamente economías liberales.

Téase en este sentido los trabajos presentados a la Conferencia sobre Experiencias y lecciones de las economias pequeñas y abiertas. Instituto de Economía de Ia Universidad Católica de Chile. 1981. Particularmente los estudios de Ios profesores Pang Eng Fong (Singapur) y Chong-Xah-Lim.

'Véase, por ejemplo, Fernando Fajnzylber: "Reflexiones sobre la industrialización exportadora del sudeste asiático". Revista de la CEPAL. Diciembre de 1981. 
En la región latinoamericana se encuentran políticas de esta naturaleza, con ciertas variaciones, principalmente en el cono sur del continente. Argentina, Chile y Uruguay han adoptado políticas liberales. Brasil ha pasado por un período de política liberal, Pero ha regresado en ciertos aspectos a una economía de intervención estatal. Bolivia, Colombia y Perú también han comenzado su aproximación gradual a una política económica liberal.

$\mathrm{El}$ caso de Chile ofrece quizás Ios resultados más espectaculares, durante el período que comprende los últimos cinco años. Después de haber experimentado un crecimiento negativo, que superó el $10 \%$ en el período 1972-1974, se inició una rápida recuperación, para llegar a un promedio positivo de $8 \%$ en $1977-1979$ y nivelarse en un $6.5 \%$ posteriormente. En algunos años, particularmente en 1977, el crecimiento superó el $9 \%$ anual. El proceso inflacionario se redujo de un $500 \%$ en 1974 a un $9.5 \%$ en 1981. Las reservas internacionales pasaron de 138 millones de dólares en 1977 a 4.000 millones de dólares en 1981. Las exportaciones han crecido de 3.000 millones de dólares en 1977 a cerca de 5.000 millones en 1980, con especial énfasis en exportaciones diferentes del cobre, producto que ha ido disminuyendo su incidencia porcentual en el total de las exportaciones. Las importaciones han crecido igualmente de 3.126 millones de dólares en 1977 a cerca de 6.000 millones en 1980. También cabe indicar que el Producto Nacional Bruto pasó de 18.745 millones en 1977 a 244.551 millones en $1981^{9}$ A partir de 1982 se espera una baja relativa en estos porcentajes, como consecuencia de los efectos derivados de la recesión internacional.

\section{El intercambio comergial entre América litina y asean}

Las cifras disponibles para 1980 indican que el comercio total entre América Latina y ASEAN ha sido aproximadamente 1.000 millones de dólares. ASEAN exportó ese año hacia la región latinoamericana 663 millones de dólares, que representan el $1 \%$ de sus exportaciones totales. Por su parte, América Latina exportó hacia ASEAN 342 millones, equivalentes al $0,4 \%$ de sus exportaciones totales. Si bien como porcentaje estas cifras son todavía pequeñas - lo que se explica por ser el inicio de este intercambio muy reciente-, los volúmenes totales no dejan de ser significativos. Además, se trata de una tendencia creciente, por lo que cabe esperar cifras todavía mayores en el futuro cercano ${ }^{10}$.

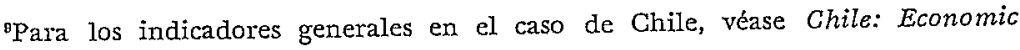
Report. Bнс. 1980.

rovéase Francisco Orrego Vicuña y Juan Reutter: Economic Cooperation and Conflict in the Pacific Basin: a South American View. Pacific Forum Symposium. Bangkok. November 14-15, 1981. 
Cabe observar; - desde luego, que cada uno de estos grupos regionales es altamente dependiente en su comercio exterior de Japón y de Ios Estados Unidos, Io que también ha limitado una mayor expansión de su comercio recíproco. Argentina, Brasil, 'Ghile y Cuba. han sido los mayores exportadores latinoamericanos hacia ASEAN, en tanto que Singapur representa el $77 \%$ de las exportaciones de AsEAn hacia América Latina. También Malasia ha comenzado a surgir como un país significativo, concentrando el $17 \%$ de las exportaciones de ASEAN hacia América Latina. Es interesante, asimismo, señalar que las exportaciones totales de cada región son comparables, pues América Latina en 1980 alcanzó un total de $82.392 \mathrm{mi}-$ llones de dólares y ASEAN la cifra de 67.063 millones.

En el plano comercial también debe tenerse presente el caso de Ios países en desarrollo más avanzados de Asia y de América Latina que se han indicado anteriormente, lo que permite también apreciar interesantes perspectivas ${ }^{11}$. Las exportaciones de este grupo asiático hacia América Latina han crecido un $23,8 \%$ anual en el período $1969-1978$ y un 13,8\% anual en el período 1974-1978, que es el último disponible con cifras completas. A este respecto, debe hacerse notar el caso especial de Chile, que durante este último período aumentó sus importaciones desde este grupo en un $66 \%$ anual.

Respecto de este grupo, los principales importadores latinoamericanos son Panamá $(35 \%)$ - particularmente en función de los servicios-, Brasil (9\%), México y Chile $(7 \%)$. A su vez, los principales exportadores latinoamericanos al grupo son Brasil (45\%), Argentina $(18 \%)$ y Chile $(8 \%)$, todo ello siempre de acuerdo a las cifras señaladas de 1978. Esta misma comparación indica que Taiwán absorbe el 42\% de las importaciones desde América Latina, en tanto que Singapur el $25 \%$ y Corea el $15 \%$. Los principales exportadores del grupo asiático son Taiwán (34\%) y Singapur (24\%).

Las principales exportaciones de los países asiáticos de este grupo que se comenta son el vestuario, productos del petróleo, caucho, maquinaria eléctrica, aceites vegetales, equipos de comunicación, petróleo crudo, estaño y otros productos de menor significación. En el caso latinoamericano, las principales exportaciones son el petróleo crudo, azúcar, hierro, café, cobre, nueces y semillas de aceite, alimentos para animales, maíz y otros productos principalmente agrícolas y minerales.

Interesa finalmente indicar algunas cifras específicas del comercio entre Chile y los países de ASEAN, correspondientes a 1980, las que también muestran una tendencia sostenida al crecimiento. EI total del intercambio alcanzó a 36,97 millones de dólares ese año,

11Véase Ernesto Tironi: Trade Relations between Latin America and the Asian Advanced Developing Countries. Eleventh Pacific Trade and Develop-
ment Conference. Seoul. September . 1-4, 1980. Mimeo. 
de los cuales 11,99 millones fueron exportaciones chilenas hacia ASEAN y 24.98 millones importaciones por Chile desde ASEAN. Ello arrojo un saldo negativo para Chile de 12,99 millones. El comercio total con Singapur fue de 13,1 millones (3,4 exportaciones y 9,7 importaciones); con Malasia fue de 9,8 millones (0,59 exportaciones y 9,2 importaciones); con Filipinas fueron 6,8 millones $(4,2$ exportaciones y 2,6 importaciones); con Tailandia alcanzó a 4,6 millones ( 1.3 exportaciones y 3,2 importaciones); el más bajo fue Indonesia con 2,7 millones $(2,5$ exportaciones y 0,28 importaciones $)$.

\section{IMPORTANGLA DE LAS PERSPEGTIVAS COMERGIALES}

Las relaciones comerciales que se han indicado están produciendo por efecto el acercamiento de los países en desarrollo más adelantados de cada región, entre los que se cuenta Singapur, Malasia, Taiwán, Hong-Kong y Corea, por una parte, y Argentina, Brasil, Chile y México, por otra parte. Esta es la primera vez en que tiene lugar este fenómeno y de él derivan algunas consecuencias de la mayor importancia internacional. Este grupo de países en desarrollo, caracterizados por su dinamismo económico, representan hoy día más de un tercio de las exportaciones totales de los países en desarrollo del mundo entero. Hacia fines de la presente década esta participación llegará a aproximadamente al $45 \%$ de esas exportaciones totales, correspondiéndole al grupo asiático un $27 \%$, pero estimándose que las exportaciones del grupo latinoamericano también crecerán rápidamente ${ }^{12}$.

Esta situación le otorgará al grupo de 10 países en desarrollo más adelantados un papel de gran peso en las negociaciones económicas internacionales, pudiendo adquirir una influencia que hasta ahora se ha visto en cierto modo difusa. Además, se trata de paises de un rápido crecimiento, con aumentos anuales del Producto Nacional Bruto de alrededor del $8 \%$ como promedio, y con un crecimiento anual de las exportaciones del 12\%.

Estas mismas características contribuirán a acrecentar el intercambio recíproco entre los dos grupos, incluyendo desde luego el del propio ASEAN. El crecimiento del ingreso nacional y la apertura de algunos países latinoamericanos al libre comercio, también ayudarán a esta expansión de las corrientes comerciales entre las dos regiones.

Dentro de esta perspectiva es interesante examinar la composición del intercambio recíproco, aspecto que permite apreciar ciertos factores que también son positivos para estimular el crecimiento comercial. Las exportaciones de los países asiáticos hacia América Latina consisten principalmente en productos manufacturados

12Tbid. 
y sólo secundariamente en productos primarios. En 1977 estas cifras fueron, respectivamente, de 298 millones de dólares y de 193 millones de dólares, de acuerdo a estudios realizados para un grupo de países asiáticos representativos. En cambio, si se analiza la composición de las exportaciones latinoamericanas al Asia, se podrá observar la relación inversa, esto es, se trata principalmente de productos primarios y sólo secundariamente de productos manufacturados. Las cifras para el mismo año fueron, respectivamente, de 512 a 66 millones de dólares. La incidencia de las manufacturas en las ventas externas de los países asiáticos es cuatro veces mayor que en el caso de los países latinoamericanos.

Como se ha indicado anteriormente, los principales rubros de exportación latinoamericana consisten en trigo, carne, azúcar, alimentos para animales, vegetales, papel, celulosa y fertilizantes. Los países asiáticos exportan principalmente barcos, vestuario, equipos de telecomunicación y maquinaria eléctrica.

De esta manera, es posible concebir una importante complementariedad entre las economías de los países más avanzados de cada grupo, lo que también es un estímulo para el crecimiento comercial. En el caso de ASEAN, sin embargo, la complementariedad se aplica más bien a Singapur y Malasia, pues las producciones de los otros miembros son en general competitivas con aquellas de América Latina, sobre todo en petróleo, calzado, café y algunos minerales. Pero aún en este caso, es necesario separar a Chile y Argentina dél resto de América Latina, pues se trata de países de producción templada, que también es complementaria de la producción tropical que caracteriza a Filipinas, Indonesia o Tailandia. Se puede apreciar así que el factor complementariedad se encuentra presentè en un buen número de situaciones, sin excluir por cierto enteramente los casos de competencia ${ }^{13}$.

\section{FORMAS NEGESARIAS DE GOOPERACTÓN}

El hecho de que los países más avanzados de las dos regiones compartan una similar posición en la economía internacional, sugiere la posibilidad de desarrollar formas de cooperación que faciliten el tratamienta de problemas que son comunes. Si se piensa, por ejempio, en las dificultades suscitadas por el proteccionismo de algunas grandes potencias industriales, que afecta gravemente las exportaciones de aquellos países que han fundado su modelo de desarrollo en el dinamismo de su sector exportador, podrá apreciarse que tanto los países de ASEAN y otros de Asia, como los países de Amé-

${ }^{13} \mathrm{Un}$ análisis periódico de las relaciones comerciales puede encontrarse en Chile R'eport for ASEAN countries, publicado por la Embajada de Chile en Singapur. No I. Nov-Dec. 1981 y siguientes. 
rica Latina tienen un interés especifico en buscar formas que eviten este tipo de medidas adversas ${ }^{14}$.

En este sentido, los aspectos relativos a la liberalización del comercio internacional debieran interesar de manera prioritaria a los dos grupos de países, los cuales podrían proceder en muchas materias de común acuerdo. El tema de la reforma del Gatr y la conferencia ministerial de las partes contratantes convocadas para 1982, proporcionará una importante oportunidad en este sentido. Además, en alguna medida otros páses, como Australia y Nueva Zelandia, también comparten problemas de esta naturaleza, con lo cual podría incrementarse la capacidad de negociación y presión de los países afectados.

Por otra parte, también es necesario tener presente que este grupo de países en desarrollo podría sufrir una importante competencia de parte de algunas economías emergentes de Asia ${ }^{15}$. Asi, por ejemplo, si un país como China desarrollara su capacidad exportadora sobre la base de asociarse con grandes conglomerados multinacionales y ofrecer bajos costos de producción y mano de obra, posiblemente la participación de ese grupo asiático y latinoamericano en el comercio mundial se vería disminuída. Igualmente en el campo de la energía es necesario observar que las exportaciones de petróleo chino son competitivas con la de países como Indonesia y México. Se ha estimado que para el año 2000 el comercio de carbón aumentará en cuatro veces, siendo Chile un importante productor. de este mineral. Todo ello sugiere igualmente formas de cooperación.

EI creciente comercio entre ASEAN y América Latina está también llevando a formas de integración horizontal y vertical. Entidades de Singapur han realizado, por ejemplo, importantes inversiones agricolas en Chile para la producción de frutas templadas. También se han suscrito acuerdos de joint-venture entre grupos empresariales de 'Chile y Singapur, para la promoción del comercio entre las dos regiones. Los vínculos financieros han comenzado igualmente a desarrollarse, tanto por medio de la emisión de bonos y la suscripción de créditos en Singapur por empresas chilenas, como por la apertura de sucursales bancarias de Chile en Singapur y de Hong-Kong en Chile, entre otras expresiones.

Entre los problemas que requieren urgentemente de soluciones se encuentra el de la información y el transporte, para lo cual tam-

${ }^{14}$ Véase estudio cit. Nota 10 supra.

16Véase en general, Juan Reutter: "Diagnóstico y perspectivas de las relaciones económicas entre la región Asia Pacífico y América Latina". En Comunidad del Pacifico. Vol. 1. 
bión se ha comenzado a desarrollar la necesaria cooperación. El desarrollo de la información comercial y financiera permitiría incrementar sostenidamente el intercambio. Empresarios chilenos han propuesto la creación de un centro de información de este tipo para Ios países del Pacífico, en el ámbito del Pacific Basin Economic Council, entidad que se reunirá en Chile en 1983, por primera vez en un país latinoamericano.

El transporte ha sido otro obstáculo difícil de superar, incidien do de manera negativa en los costos del comercio recíproco. Tradicionalmente era necesario utilizar la ruta del Pacífico Norte, pero recientemente han comenzado a establecerse servicios directos. Una compañía chilena mantiene servicios directos con Singapur e indirectos a través de Hong-Kong y Tokyo, habiendo celebrado acuerdos con una empresa de Singapur. Algunos servicios europeos también han comenzado a operar. De la misma manera, próximamente se iniciará un servicio directo entre Sidney y Valparaíso. Singapur, Manila, Papeete y Panamá han comenzado a operar como puertos de transbordo.

En materia de servicios aéreos todavía existen varios obstáculos. La única ruta a través del Pacífico Sur es atendida por Lan-Chile hasta Papeete, y ocasionalmente hasta Fiji. Varios planes de extensión hacia el Sudeste asiático se han visto impedidos por la actitud proteccionista seguida por Australia, siendo su mercado decisivo para justificar esas nuevas rutas. Chile y Singapur han suscrito recientemente un convenio aéreo, reafirmando la política de cielos abiertós que ambos países practican. Sus respectivas aerolíneas han experimentado dificultades similares en el mercado australiano.

Esta comunidad de intereses que se ha ido creando entre las dos regiones, que comparten las políticas, oportunidades y problemas de los llamados Newly Industrialized Countries (NIC's), grupo que también ha sido identificado como una Glase Media de las Naciones ${ }^{16}$, es el que explica las múltiples formas de cooperación que son posibles, y las cuales recién han comenzado a manifestarse. En la medida en que esta cooperación se concrete, los países participantes adquirirán una capacidad efectiva de liderazgo internacional.

\section{LA POLÍtTCA DE REGURSOS NATURALES}

Una de las áreas donde también se observan oportunidades importantes de cooperación es en la política relativa a los recursos naturales. En algunos casos se trata de formas de cooperación funcio-

${ }^{1}$ Francisco Orrego Vicuña: América Latina. ¿Clase Media de las Naciones? Instituto de Estudios Internacionales de la Universidad de Chile. 1979. 
nal, que se refieren a algunos recursos en particular. Este es, por ejemplo, el caso de Consejo Intergubernamental de Países Exportadores de Cobre (CIPEC), en que participan Chile y Perú, como también Indonesia, Filipinas y Papúa Nueva Guinea, además de otros países. Otra experiencia interesante en este sentido es la del Acuerdo del Estaño, que reúne a Bolivia y Malasia como dos im. portantes productores. En el sector energético se observan igualmente algunas formas de cooperación, participando países de una y otra región en instituciones comunes.

En otros casos se trata de la política de recursos naturales en un sentido más amplio. El campo general del derecho del mar ofrece un destacado ejemplo de cómo ha sido posible y efectiva la cooperación entre Ios países de ASEAN y América Latina. En el ámbito de la Conferencia de las Naciones Unidas sobre el Derecho del Mar -actualmente presidida por el distinguido embajador de Singapur, señor T. T. B. Koh-, se han negociado numerosos problemas de interés común para las dos regiones, habiendo los respectivos países obrado normalmente de acuerdo a una política y posición común en estas materias.

La aprobación de la Zona Económica Exclusiva -institución que por primera vez en el mundo fue aplicada por Chile a partir de 1947-, contó con el apoyo unánime de los países de ASEAN. Igual coincidencia de puntos de vista puede apreciarse en materias como el régimen de la pesca, las normas aplicables a los archipiélagos. oceánicos -como Filipinas e Indonesia- o el régimen relativo a la exploración y explotación de los recursos minerales de los fondos marinos, con particular referencia a la necesidad de prevenir efectos adversos para los productores terrestres de los mismos minerales, entre los que se cuentan Chile, Perú, Indonesia y Filipinas.

También cabe observar que las principales rutas de acceso al Océano Pacífico son controladas por países de ASEAN y América Latina. Los Estrechos de Malaca están bajo la juxisdicción de Indonesia, Malasia y Singapur; el Estrecho de Magallanes se encuentra íntegramente rodeado por territorio chileno; y el Canal de Panamá lleva el nombre de ese país. Al respecto, podría desarrollarse un valioso intercambio de experiencias en cuanto a la administración de estas vías oceánicas, con particular referencia a los problemas de navegación, contaminación y esquemas de separación de tráfico, entre otras cuestiones. El establecimiento de un Comité Goordinador podría ser una medida conveniente ${ }^{17}$.

${ }^{1 T F r a n c i s c o ~ O r r e g o ~ V i c u n ̃ a: ~ " E l ~ P a c i f i c o ~ i n s u l a r ~ e n ~ u n a ~ p e r s p e c t i v a ~ l a t i n o a m e-~}$ ricana". En Comunidad del Paclfico. Vol, 1. 


\section{EL MARCO POLÍTICO DE LA GOOPERAGIÓN}

En algunos casos y situaciones se pueden observar también ciertas identidades $0^{\circ}$ coincidencias de carácter político, que se reflejan en algunas formas de entendimiento o cooperación entre países de ASEAN -y América Latina. Históricamente, el caso más evidente es el de las Filipinas, por compartir los mismos orígenes coloniales con América Latina. El rol de la Iglesia en ese país es, por ejemplo, muy similar al que puede observarse en muchos países latinoamericanos. La rápida comunicación de los grandes movimientos y tesis intelectuales, con sus expresiones políticas, religiosas o de otra índole, es característica de una cierta comunidad entre las Filipinas y algunos países de nuestra región. No por casualidad es que en el caso de varios organismos económicos internacionales, las Filipinas participan en el mismo grupo con los países de América Latina.

En el marco contemporáneo también han surgido identidades de tipo político que deben tenerse en cuenta. La existencia de gobiernos fuertes, que en algunos casos corresponden al modelo autoritario, es un fenómeno común en las dos regiones ${ }^{18}$. En varios casos ello se explica por las difíciles experiencias vividas en la política interna, de las cuales no ha estado ausente la existencia de focos guerrilleros, la lucha ideológica o formas de enfrentamiento directo. Similares situaciones y características se observan también en otros países asiáticos, como Corea y Taiwán.

Estos factores han incidido en el desarrollo de algunas percepciones comunes en el campo de la política internacional. Las preocupaciones en materia de seguridad regional son evidentes en las dos regiones ${ }^{19}$. Aun cuando ASEAN no es una alianza de carácter militar, los países miembros manifiestan una natural inquietud por sus problemas defensivos ${ }^{20}$, al igual que ocurre en el caso latinoameri: cano, donde los mecanismos existentes en materia de seguridad continental han dejado de ser eficaces o de merecer la confianza de los países de la región, principalmente por las cambiantes políticas seguidas por los Estados Unidos durante la década pasada. Parte de esta inquietud se debe, en el caso de ambas regiones, a la amenaza que representan algunas potencias, y a los problemas de equilibrio de poder.

Sobre la base de esta común percepción de la política internacional, han surgido posiciones también comunes respecto de algunos organismos y conflictos. Un ejemplo interesante es el del movi-

\footnotetext{
${ }^{18}$ Robert Bosa: "Les regimes autoritaires de droite en Asie Orientale". Project. Paris, No 126. Juin 1978.

${ }^{10}$ Para el caso de ASEAN, Lau Teik Soon: "ASEAN development and concept of a Pacific Community". En Comunidad del Pacifico. Vol. 1.

${ }^{2}$ Rosewati Ismail: "ASEAN in the cooperation and the security of the AsiaPacific region". En Comunidad del Pacífico. Vol. 1.
} 
miento de los países no alineados, en el que participan varios países de una y otra región. Cuando este movimiento abandonó sus objetivos iniciales de auténtico no alineamiento, y pasó a servir los intereses de una determinada potencia, fueron los países miembros de ASEAN y de América Latina los que iniciaron un esfuerzo destinado a corregir esa idesviación y asegurar que se mantuviera la equidistancia respecto de los grandes bloques tradicionales. En gran medida esta rectificación ha sido exitosa.

Otro caso importante en que se manifiesta esta percepción común es el del conflicto de Kampuchea. La posición que han adoptado los países de ASEAN en esta materia, exigiendo el retiro de Vietnam, ha sido ampliamente compartida por países de América Latina, según pudo apreciarse en la Conferencia especial de las Naciones Unidas sombre Kampuchea. El principio de no intervención, el respeto del derecho internacional y la solución pacífica de controversias, son algunos de los valores que han llevado a ASEAN y América Latina a adoptar una posición común en este caso. Ello abre las perspectivas de futuras formas de cooperación destinadas a salvaguardar estos y otros valores fundamentales de la comunidad internacional.

\section{Asean, Amértca latina y la perspectiva de la COOPERAGTón EN EL PaGífico}

Recientemente, el Gobierno del Japón ha planteado algunas iniciativas preliminares destinadas a explorar la idea de la creación de una Comunidad del Pacífico, concepto que posteriormente ha interesado especialmente a algunos medios australianos ${ }^{21}$. Esta idea ha sido considerada con atención por algunos países latinoamericanos, en cuanto ella pudiera significar un incremento de la cooperación en el Pacífico, pero sujeta, sin duda, a ciertas indispensables precisiones. En este sentido, la reacción ha sido una mezcla de interés y cautela. Similares enfoques han emanado de las instituciones académicas de los países de ASEAN $^{22}$.

En este plano, el intercambio de opiniones entre los países de ASEAN, el Pacífico Sur y América Latina, que es el núcleo de los países en desarrollo eventualmente llamado a participar en un esquema de cooperación transpacífico, podría resultar decisivo para asegurar que toda iniciativa en este campo tenga en cuenta el interés fundamental de este grupo. A la vez, se evitaría que pueda intentarse dividir la opinión de dichos países.

${ }^{21}$ Sir John Crawford (ed.): Pacific Economic Cooperation. Heineman Educational Books. Singapur, 1981.

${ }^{22}$ ASEAN and the Pacific Community. A report prepared by Akrasanee et al, Nay 1981. 
La coordinación que se ha postulado en este artículo entre los países en desarrollo de la Cuenca del Pacífico podría crear las bases para una sólida cooperación a través del Pacífico, fundamentada en la trilogía de ASEAN, el Pacífico Sur y América Latina. Este puente de acción transpacífico influiría positivamente en que una eventual Gomunidad del Pacífico fuera más estable en cuanto aseguraría una mayor participación, más equitativa desde el punto de vista de las necesidades del desarrollo económico y, en definitiva, más equilibrada como concepción. Además, se tendría la seguridad de que un posible esquema de cooperación de esta naturaleza no afectaría las modalidades de cooperación que cada región deseara desarrollar entre sus países miembros, como tampoco dificultaría las relaciones que se deseen mantener con cualquier otro país o región del mundo. La cooperación en el Pacífico sería así complementaria y no sustitutiva de los enfoques prevalecientes en la actualidad.

La presencia contemporánea de América Latina en la cooperación transpacífico se inició recién hace una década, cuando el Instituto de Estudios Internacionales de la Universidad de Chile convocó en 1970 a una Conferencia internacional sobre el tema "América Latina vuelve al Pacífico"23. Entre otras personalidades, esa Conferencia contó con la participación del señor S. Rajaratnam. En octubre de 1981 el Instituto recibió la visita de otra distinguida personalidad de ASEAN, el señor Ministro de Relaciones Exteriores de Tailandia, Siddhi Savetsila. Ella sirvió para apreciar restropectivamente los progresos logrados durante la década de 1970 en este campo, en lo que se observa un incremento sostenido del conocimiento, la cooperación y el intercambio recíproco. Esta es una realidad que deberá traducirse cada día en pasos más específicos. $\mathrm{Pa}$ ra ello, la visión y la relación entre las principales instituciones académicas de las dos regiones será un factor decisivo del progreso futuro de la cooperación transpacífico,

${ }^{23}$ Para un informe sobre las actividades del Instituto de Estudios Internacionales de la Universidad de Chile en relación al Pacifico, véase Project on the Pacific, 1982. 